\title{
THE MARKOV MODEL AS A PATTERN FOR EARTHQUAKES RECURRENCE IN SOUTH AMERICA
}

\section{T.M. TSAPANOS ${ }^{1}$}

\begin{abstract}
The well known stochastic model of the Markov chains is applied in south America, in order to search for pattern of great earthquakes recurrence. The model defines a process in which successive state occupancies are governed by the transition probabilities pij, of the Markov process and are presented as a transition matrix say $\mathrm{P}$, which has $\mathrm{NxN}$ dimensions. We considered as states in the present study the predefined seismic zones of south America. Thus the visits from zone to zone, which is from state to state, carry with them the number of the zone in which they occurred. If these visits are considered to be earthquake occurrences we can inspect their migration between the zones (states) and estimate their genesis in a statistical way, through the transition probabilities. Attention is given in zones where very large earthquakes with $\mathrm{Ms} \geq 7.8$ have occurred. A pattern is revealed which is suggested migration of these large shocks from south towards north. The use of Monte Carlo simulation verify the defined pattern.
\end{abstract}

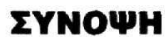

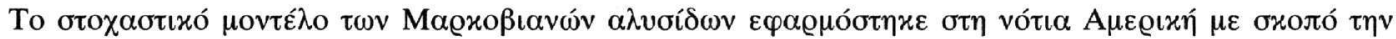

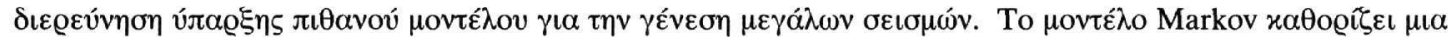

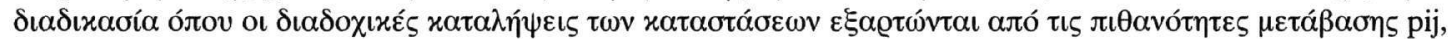

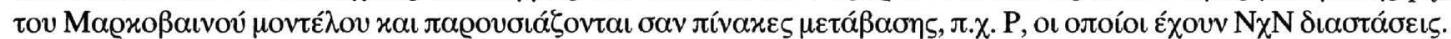

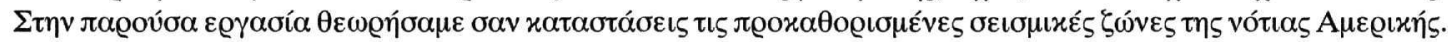

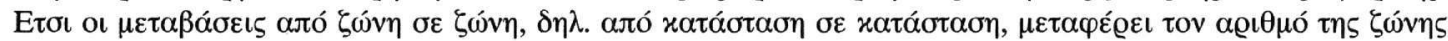

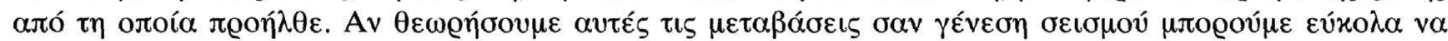

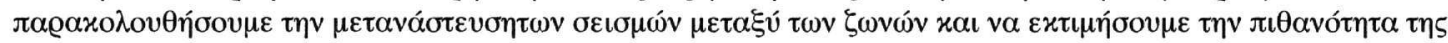

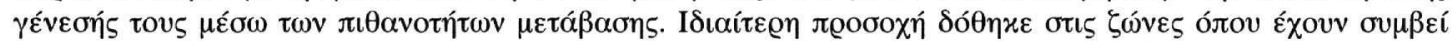

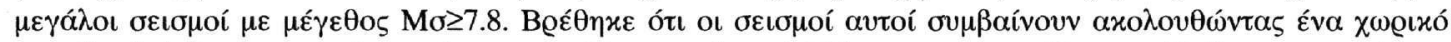

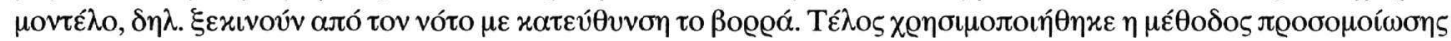

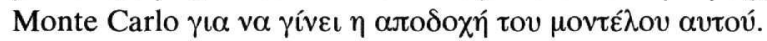

KEY WORDS: Markov model, transition probabilities, south-north migration pattern, south America.

\section{INTRODUCTION AND DATA USED}

The most common model for earthquake occurrence is the Poisson one which assumes spatial and temporal independence of all earthquakes including great events. For example the occurrence of one earthquake does not affect the likelihood of a similar earthquake at the same location in the next time unit.

Among other models, Markov chain is an alternative probabilistic model of earthquake recurrence, with various applications. The Markov process has a basic property which is the memory of one-step. According to this property the probability of being in some state $\mathrm{j}$ at a future time is deduced from the knowledge of the state $i$ at an earlier time $t$ and is independent of the history of the process up to time $t$ (Anagnos and Kiremidjian, 1988). Vere-Jones (1966) and Knopoff (1971) applied the continuous-time and continuous-state Markov process to describe aftershock sequences as well as sequences of main events followed by aftershocks, respectively. Vagliente (1973) proposed a two-state

Markov-chain for earthquake occurrence in specific time interval. Lomnitz-Adler (1983) used a simulation of Markov model to give a simplified representation of the spatial distribution of earthquakes on adjacent faults. In such formulation the energy or stress levels constitute the states of the process. Steps from one state to 
another imply the occurrence of earthquakes and are described by the transition probabilities (Anagnos and Kiremidjian, 1988).

The time span covered in the present study is 1899-1992. For this purpose a catalogue of events was constructed taking into account the earthquakes listed in the catalogues of: a)Gutenberg and Richter (1954), b) Rothe (1969) and c) the ISC bulletins from 1964 up to 1992. This catalogue is improved considering the magnitudes given by Pacheco and Sykes (1992). The present study is restricted to strong ( $\mathrm{M} \geq 6.5)$ and shallow ( $\mathrm{h} \geq 60 \mathrm{~km})$ earthquakes. Often such earthquakes are responsible for heavy damages and casualties.

In the present study a methodological proposal is made in order to observe the spatial distribution of the earthquakes in south America, searching in this way for a pattern for shocks recurrence. The pattern can easily revealed following the transition probabilities obtained through the Markov process.

\section{METHOD APPLIED}

Markov models are useful in describing a unique type of dependence in a sequence of events. For these models a state space $\mathrm{E}=\{1,2,3, \ldots, \mathrm{N}\}$ is defined such that the state may corresponds to various quantities. This process $\{X(t), t>0\}$ describes the visits to these states which is the Markov-chains process. Anything can be considered as a state in the Markov model, i.e. stress or energy release levels or magnitudes of earthquakes, etc. In the present work as states are defined the seismic zones in which the investigated area is divided, following a modified version of Papadimitriou (1993) zonation, and is coincided with the zones defined by Galanis (2001). The zones which are considered are six. So we have 6 states.

Let pij be the probability that Markov process, which entered state $i$ on its last transition, will enter state $j$ on the nest transition and is computed by the maximum likelihood estimators. The transition probabilities which completely determine the Markov process must satisfy the following properties:

$$
\begin{aligned}
& p_{i j} \geq 0 \quad i=1,2,3, \ldots N \quad j=1,2,3, \ldots N \\
& \text { and } \\
& \sum_{j=1}^{N} p_{i j}=1
\end{aligned}
$$

where $\mathrm{N}$ is the total number of states in a system.

Whenever the process enters a state $i$ the likelihood that it will go to state $j$ in the next step is determined by the transition probability pij, which can be estimated by:

$$
p_{i j}=\frac{n_{i j}}{n_{i}}
$$

where nij is the observed number of the transitions from state $\mathrm{i}$ to state $\mathrm{j}$ and $\mathrm{nI}$ is the observed number of transitions from state $\mathrm{i}$ to all possible states.

The frequency of visits in every state and the transition probabilities between the states can be expressed through the transition probability matrix. A frame work of a transition matrix $(2 x @)$ is presented below:

$$
\text { state } 1 \quad \text { state } 2 \text { row total }
$$

$\begin{array}{cccc}\text { state 1 } & \mathrm{n}_{11} & \mathrm{n}_{12} & \mathrm{n}_{1} \\ & \mathrm{p}_{11} & \mathrm{p}_{12} & \\ \text { state 2 } & & & \\ & \mathrm{n}_{21} & \mathrm{n}_{22} & \mathrm{n}_{2} \\ & \mathrm{p}_{21} & \mathrm{p}_{22} & \end{array}$

In this matrix, the frequency of transitions in every state and the transition probabilities between the states, are demonstrated. Symbols $\mathrm{n}_{11}, \ldots, \mathrm{n}_{22}$ denotes frequency of visits in every state, while $\mathrm{p}_{11}, \ldots, \mathrm{p}_{22}$ are the corresponding transition probabilities of visits from state $i$ to state $j$ and $n_{1}$ and $n_{2}$ represent the total number of visits in every row. 


\section{APPLICATION OF THE MODEL: A PATTERN FOR THE SPATIAL DISTRIBUTION OF THE EARTH- QUAKES}

The area of south America is one of the most seismically active regions of the world. It is bounded between the latitudes $47^{\circ} \mathrm{S}-0^{\circ}$ and longitudes $85 \mathrm{o}-65 \mathrm{oW}$. The spatial distribution of earthquakes in the examined area and the number of each zone (state) is depicted in Figure (1). The whole are has experienced severe and catastrophic earthquakes $(M \geq 7.8)$. Six of them (with $M \geq 7.8)$ occurred in the present century. Temporally they occurred in 1906 with M-8.0, in 1922 with $M=8.1$, in 1942 with M-8.0 and the largest of the present century in 1960 with $M=8.5$. two events equal to 7.8 occurred in 1906 and 1985. The places (zones-states) of their occurrence are illustrated in Figure (2).

The assumption made is that, if every one of the examined zones is considered to be a state in the Markovprocess, then every shock which occurs in each one of them will be characterized by the number of the zone. Then we can check the spatial occurrence of strong earthquakes in a quantitative way because the visits (which are considered as occurrence of earthquakes) from on state to another can be expressed through the transition probabilities. The frequency of visits in every zone and the transition probabilities are presented in Table (1).

Table 1. Frequency of visits (earthquake occurrence) and the transition probabilities (in parenthesis) of strong

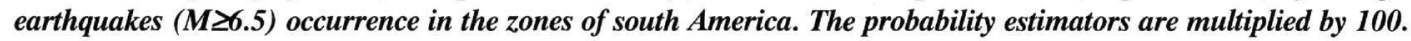

$\begin{array}{cccccccc} & 1 & 2 & 3 & 4 & 5 & 6 & \text { row total } \\ 1 & 1(7.1) & 1(7.1) & 2(14.3) & 4(28.6) & 4(28.6) & 2(14.3) & 14 \\ 2 & 1(10.0) & 1(10.0) & 1(10.0) & 3(30.0) & 3(30.0) & 1(10.0) & 10 \\ 3 & 4(22.2) & 1(5.5) & 3(16.7) & 3(16.7) & 3(16.7) & 4(22.2) & 18 \\ 4 & 3(15.0) & 3(15.0) & 7(35.0) & 2(10.0) & 2(10.0) & 3(15.0) & 20 \\ 5 & 2(12.5) & & 3(18.8) & 4(25.0) & 3(18.8) & 4(25.0) & 16 \\ 6 & 3(16.7) & 4(22.2) & 2(11.1) & 2(11.1) & 2(11.1) & 5(27.8) & 18\end{array}$

A careful look in Table (1) shows that zones 1,2,3 have high transition probabilities for a north-south migration of earthquakes with $M \geq 6.5$. This is in accordance to Kelleher's (1972) observations, who suggested a northsouth migration in the same area. On the other hand zones 4,5,6 demonstrate a tendency for a south-north migration. More clear observations are illustrated when we consider only large ( $M \geq 7.0)$ earthquakes. In Table(2) we tabulated the frequency of visits, as well as the transition probabilities in every zone.

Table 2. Frequency of visits (earthquake occurrence) and the transition probabilities (in parenthesis) of large

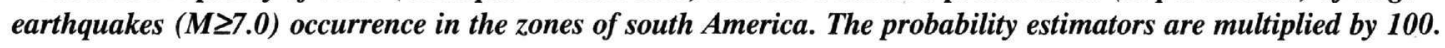

$\begin{array}{cccccccc} & 1 & 2 & 3 & 4 & 5 & 6 & \text { row total } \\ 1 & & & 1(20.0) & 2(40.0) & 1(20.0) & 1(20.0) & 5 \\ 2 & & 1(16.7) & & 2(33.3) & 3(50.0) & & 6 \\ 3 & 1(12.5) & & 1(12.5) & 2(25.0) & 1(12.5) & 3(37.5) & 8 \\ 4 & 1(7.1) & 2(14.3) & 4(28.6) & 2(14.3) & 2(14.3) & 3(21.4) & 14 \\ 5 & & & 2(25.0) & 3(37.5) & 2(25.0) & 1(12.5) & 8 \\ 6 & 3(23.1) & 4(30.7) & 1(7.7) & 1(7.7) & 2(15.4) & 2(15.4) & 13\end{array}$

We can conclude from Table (2) that zone 1 (state 1) is most likely to move into zone 4, while zone 2 shows a preference to visit zone 5. High transition probabilities are observed of the order of $40.0 \%$ and $50.0 \%$, respectively. High (37.5\%) transition probability is shown when zone 3 moved to zone 6 . Also probabilities less than $40 \%$ but still higher than others in the row are observed for the zones 4, 5 and 6 . A transition probability (28.6\%), which is distinguished from the other ones, is demonstrated when zone 4 visits zone 3 . Steps of zone 5 to zone 4 and of zone 6 to zone 2 are observed with high transition probabilities, which are $37.5 \%$ and $30.7 \%$, respectively. It is important that one and only one distinguish transition probability value corresponds to a particular visit, for earthquakes with $\mathrm{M} \geq 7.0$.

We remind that, we seek for a pattern of spatial distribution of large $(M \geq 7.0)$ and very large $(M \geq 7.8)$ earthquakes in the area. The number of shocks having magnitudes $M \geq 7.8$ is very limited for a Markov process modeling. However, we can assume that the conclusions derived for earthquakes with $\mathrm{M} \geq 7.0$ are valid and for the largest 
events $(\mathrm{M} \geq 7.8)$ as well. We also observed that the total number of the steps (visits) in Table (2) is not large enough, 54 transitions are counted.

For these reasons the Monte Carlo simulation technique is applied. The essence of the Monte Carlo approach is very straightforward. Since the seismic source model describes as completely as possible, both spatially and temporally, the way in which earthquakes occur in a region, it is a fairly straightforward matter to use the model to generate synthetic i.e. random Markov's chains trajectory, using the matrix of probabilities which we have here obtained as an estimate from the real data. Although this is not a new method, it is very useful in cases where the number of the data are poor. A sample with length of 10000 trials (the trial includes 54 time steps) is considered for obtaining the synthetic transition matrix of the Markov model for the 54 earthquakes tabulated with magnitudes $\mathrm{M} \geq 7.0$.

Table (3) is a synthetic matrix which presents the estimations through Monte Carlo realizations of the Markov chain. This is the mean matrix of transition probabilities for 10000 trials. Every cell in this matrix contains a reliable estimates of the probability of transition.

Table 3. A synthetic matrix (10000 steps) of transition probabilities for occurrence of earthquakes with $M \geq 7.0$ in the zones of south America. The probability estimators are presented.

$\begin{array}{ccccccc} & 1 & 2 & 3 & 4 & 5 & 6 \\ 1 & & & 0.1993 & 0.3992 & 0.2045 & 0.1970 \\ 2 & & 0.1407 & & 0.3389 & 0.5204 & \\ 3 & 0.1277 & & 0.1154 & 0.2497 & 0.1249 & 0.3824 \\ 4 & 0.0723 & 0.1441 & 0.2893 & 0.1334 & 0.1441 & 0.2166 \\ 5 & & & 0.2589 & 0.3834 & 0.2303 & 0.1273 \\ 6 & 0.2330 & 0.3164 & 0.0755 & 0.0776 & 0.1556 & 0.1419\end{array}$

The synthetic outputs from Table (3) is in very good agreement with the obtained results from Table (2). They indicated that the transition probabilities adopted for large earthquakes with magnitude $M \geq 7.0$ are valid and there is no dependence on the number of the steps, given that small number of steps are revealed in Table (2).

Now taking into account the transition probabilities of Table (2) and the occurrence of very large shocks with $\mathrm{M} \geq 7.8$ (Fig. 2) in the six zones some interesting results can be derived. An earthquake with $\mathrm{M}=8.0$ occurred in 1906 in zone 5 (state 5,33oS-72oW). According to Tables (2) the highest transition probability of $37.5 \%$, indicates a step towards zone 4 (state 4). The next earthquakes occurred in 1922 in zone 4 (state 4, 28.5oS$71.5 \mathrm{oW}$ ) and it is in accord to the probability of transition which appeared in Table (2). Visit from zone 4 (state 4) to zone 3 (state 3 ) shows transition probability of $28.6 \%$, and this comes true with the earthquake of $M=8.0$ followed in 1942 in zone 3 (state 3,14.5oS-74.8oW). We believe that, at this time, the pattern stopped going northwards. Both picks of high probability $37.5 \%$ and $25.0 \%$ (Table 2) in the row, indicated a southwards migration. Zone 3 (state 3) is most likely to move in zone 6 (state 6), with transitions probability of $37.5 \%$, where the earthquake of 1960 with $\mathrm{M}=8.5(38.1 \mathrm{oS}-73.3 \mathrm{oW})$ occurred. According to the transition probability $30.7 \%$ derived from Table (2) we expected the next earthquake occurrence in zone 2 (state 2), where in 1966 an earthquake with magnitude $\mathrm{M}=7.8(10.9 \mathrm{oS}-78.8 \mathrm{oW})$ occurred. The trend of the pattern for northwards continuation is again terminated. From zone 2 (state 2) there are high probability $50.0 \%$ (Table 2 ) for visiting zone 5 (state 5). We believe that the pattern for a south-north migration of earthquakes occurrence began again with the genesis of earthquake in 1985 with $\mathrm{M}=7.8$ in zone 5 (state $5,33.1 \mathrm{oS}-71.9 \mathrm{oW}$ ) which is in accord to the obtained transition probability. Also Comte et. al. (1986) estimated the associated rupture lengths of older events and concluded that the nearly constant repeat time for this zone is $83+7$ years. Since the earthquake of 1906 the years (79) that passed for the generation of the earthquake of 1985 are into the interval of the repeat time given by the last referred authors.

A test is made in order to check the validity of the results. The Markov process is applied in the data covered the period 1961-1992; these are the events which occurred after the 1960 earthquake in zone 6 . The data set includes events with magnitude $M \geq 6.5$. There is a transition probability of $66.7 \%$ that zone 6 will move to zone 2 . Then zone 2 with a transition probability of $50.0 \%$ steps towards zone 5 . These agree with the pattern estimated for the whole time period, as well. 


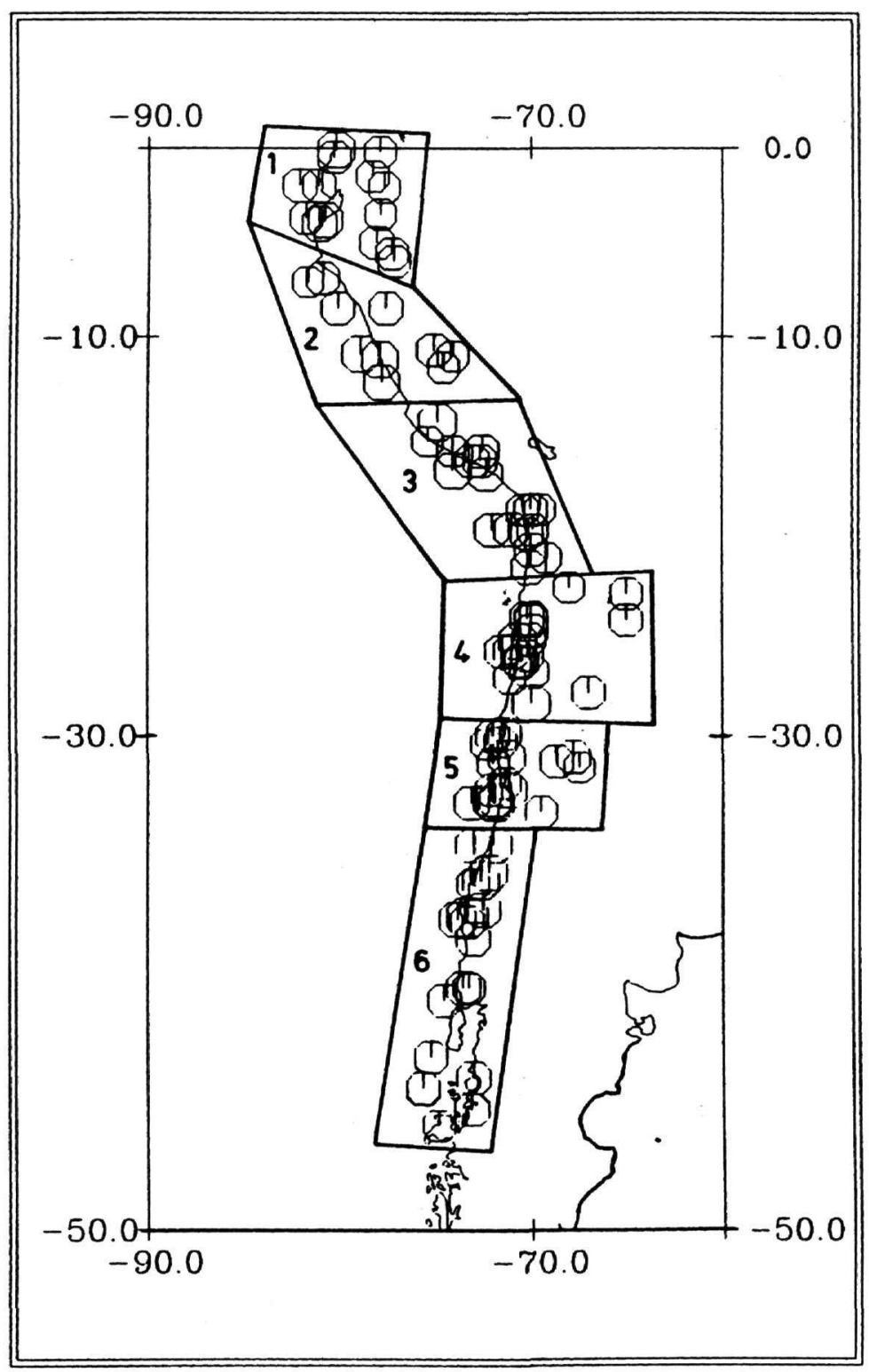

Scale 1: 5000000

Fig. 1. Spatial distribution of the epicenters of the earthquakes used in the present study. The division of the examined area into six zones (states) is also depicted

\section{RESULTS AND CONCLUSIONS}

A methodological proposal is presented in order to search for a spatial pattern of the earthquakes occurrence in South America. The Markov process is applied for this purpose and we define as states the six predetermined zones in which South America is divided. The assumption is that if every one of these zones is a state in the Markov process, then every shock which occurs in each one of them will be characterized by the number of the zone. The found pattern suggests a migration of the large ( $M \geq 7.0)$ earthquakes in a south-north direction. Because the number of the visits (steps) between the zones (states) are small (54) for these large earthquake 


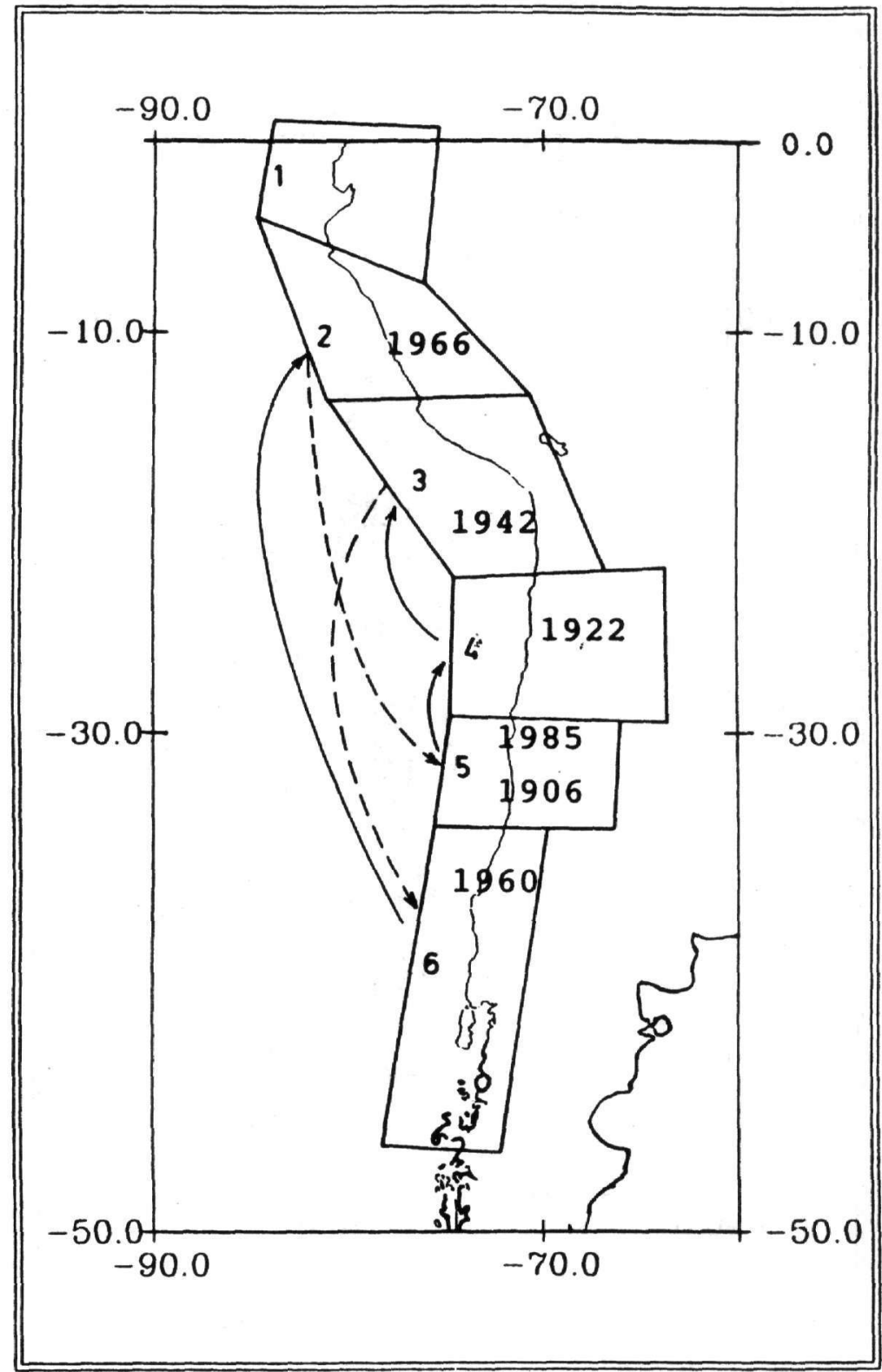

\section{Scale 1: 50000000}

Fig. 2. Sketch of the pattern found for the large earthquakes in south America. Solid arrows illustrate the southnorth direction of the pattern, while the dashed arrows show the restarting zone of the pattern. The years in which the large earthquakes occurred are depicted in the corresponding zones.

magnitudes, the efficient Monte Carlo technique is adopted, in order to check the validity of the obtained results. A set of synthetic trials is applied, consisting of 10000 steps, respectively. Comparisons between the real and the synthetic outputs manifested that the obtained transition probabilities are valid and the pattern holds for earthquakes with $M \geq 7.0$. The obtained results are in good agreement with the observations made for the six earthquakes with $M \geq 7.8$ which occurred in the whole area during the present century. The last of these earth- 
quakes occurred in $1985(\mathrm{M}=7.8)$ in zone 5 (state 5) and according the pattern there is a probability that the next shock with $\mathrm{M} \geq 7.8$ will occur in zone 4 (state 4) where the earthquake of 1922 with $\mathrm{M}=8.1$ generated. According Kelleher (1972) parts of this zone are probably under considerable tectonic strain, so the region should be considered an area of relatively high seismic risk. The conditional probabilities for this region, assuming a cycle of 104 years for the recurrence of the earthquake of 1922 , was found to be ranged between $21 \%-24 \%$ (Nishenko, 1985). The same author suggested that the event of 1922 is typical for the region and 600 to $1100 \mathrm{~cm}$ is a reliable estimate of the displacement, while the corresponding repeat times range from 74 to 135 years for an average of 104 years. According to the bulletins of USGS/NEIC an earthquake of MW=8.0 occurred in this zone $(23.34 \mathrm{oS}-70.29 \mathrm{oW}$, focal depth $45 \mathrm{~km})$ on July 30 , 1995. As it was referred three people were killed and 630 homeless. The temporal difference from the event of 1922 is 74 years which is the lowest value of repeat time interval which is suggested by Nishenko (1985). This could be considered as good test for the pattern's existence and it is obvious that the pattern "works", because the transition probability of Table (2) which is $37.5 \%$, suggested for a move from zone 5 to zone 4 . The expected place in which the next earthquake $(M \geq 7.8)$ will occur is most probably zone 3. Kelleher (1972) defined in this zone, a gap south of Lima (about $12.5^{\circ}-14.5^{\circ} \mathrm{S}$ ) which should be considered a region of relatively high earthquake risk, emphasized that no time estimate can be made for the occurrence of future major earthquake. Based on the first successful "work" of the defined pattern and in accordance with it we conclude that there is a reliable probability that the next earthquake with $\mathrm{M} \geq 7.8$ will probably strike zone 3 . The estimated transition probability $(28.6 \%)$ derived from the Table (2) strongly support this conclusion. In view of the public importance of this statement it should be mentioned that, of course, this estimate ignores the time parameter, which is needed for a most reliable earthquake prediction.

\section{REFERENCES}

ANAGNOS, T. and KIREMITZIAN, A.S. (1988). A review of earthquake occurrence models for seismic analysis, Probabilistic Engin. Mechanics, 3, 3-11.

GALANIS, O.CH. (2001). Probabilistic estimation of seismicity of the regions of Mexiko, central and south America using the Bayes statistics, Msc Dissertation (in Greek), Univ. of Thessaloniki, 97pp.

GUTENBERG, B. and RICHTER, C.F. (1954). Seismicity of the Earth and associated phenomena, Princeton Univ. Press, Princeton N.Y., 310pp.

COMTE, D., EISENBERG, A., LORCA, E., PARDO, M., PONCE, L., SARAGONI, R., SINGH, S.K. and SUAREZ, G. (1986). The 1985 central Chile earthquake: a repeat of previous great earthquakes in the region? Science, 233, 449-452.

KELLEHER, J.A. (1972). Rupture zones of large South American earthquakes and some predictions, J. Geophys. Res., 77, 2087-2103.

KNOPOFF, L. (1971). A stochastic model for the occurrence of main sequence earthquakes, Rev. Geoph. Space Phys., 9, 175-1988.

LOMNITZ-ADLER, J. (1983). A statistical model of the earthquake process, Bull. Seismol. Soc. Am., 73, 853862.

NISHENKP, S.P. (1985). Seismic potential for large and great interplate earthquakes along the Chilean and southern Peruvian margins of South America: A quantitative reappraisal, J. Geophys. Res., 90, 3589-3615.

PACHECO, J.F. and SYKES, L.R. (1992). Seismic moment catalog of large shallow earthquakes, 1900 to 1989, Bull. Seism. Soc. Am., 82, 1306-1349.

PAPADIMITRIOU, E.E. (1993). Long-term earthquakes prediction along the western coast of south and central America based on a time predictable model, Pageoph, 140, 301-316.

ROTHE, J.P. (1969). The seismicity of the Earth 1953-1965, Paris, UNESCO, 336 pp.

VAGLIENTE, V.N. (1973). Forecasting risk inherent in earthquake resistant design, Technical rep. 174, Depart. of Civil Engin., Stanford Univ., Stanford, CA.

VERE-JONES, D. (1966). A Markov model for aftershock occurrence, Pageoph, 64, 31-42. 